\title{
Chinese cities' air quality pattern and correlation
}

\author{
Wenjun Zhang ${ }^{1}$, Zhanpeng Guan ${ }^{2}$, Jianyao Li $^{2}$, \\ Zhu $\mathrm{Su}^{3, *}$, Weibing Deng ${ }^{1, *}$ and Wei $\mathrm{Li}^{1, *}$ \\ ${ }^{1}$ Key Laboratory of Quark and Lepton Physics (MOE) and Institute of Particle \\ Physics, Central China Normal University, Wuhan 430079, China \\ ${ }^{2}$ College of Physical Science and Technology, Central China Normal University, \\ Wuhan 430079, China \\ 3 National Engineering Laboratory for Educational Big Data, Central China Normal \\ University, Wuhan 430079, China \\ E-mail: suz@mail.ccnu.edu.cn, wdeng@mail.ccnu.edu.cn, \\ liw@mail.ccnu.edu.cn
}

12 February 2020

\begin{abstract}
Air quality impacts people's health and daily life, affects the sensitive ecosystems, and even restrains a country's development. By collecting and processing the time series data of Air Quality Index (AQI) of 363 cities of China from Jan. 2015 to Mar. 2019, we dedicated to characterize the universal patterns, the clustering and correlation of air quality of different cities by using the methods of complex network and time series analysis. The main results are as follows: 1) The Air Quality Network of China (AQNC) is constructed by using the Planar Maximally Filtered Graph (PMFG) method. The geographical distances on the correlation of air quality of different cities have been studied, it is found that $100 \mathrm{~km}$ is a critical distance for strong correlation. 2) Eight communities of AQNC have been detected, and their patterns have been analyzed by taking into account the Hurst exponent and climate environment, it is shown that the eight communities are reasonable, and they are significantly influenced by the climate factors, such as monsoon, precipitation, geographical regions, etc. 3) The motifs of air quality time series of eight communities have been investigated by the visibility graph, for some communities, the evolutionary patterns of the motifs are a bit stable, and they have the long-term memory effects. While for others, there are no stable patterns.
\end{abstract}

Keywords: Air quality, network, community, Hurst exponent, motif, visibility graph

\section{Introduction}

Air pollution has attracted increasing attention in recent years, due to its negative effects on the human health and environmental problems [1-3]. The relevant interesting questions include the spatial-temporal pattern of air quality, the propagation of air pollution, the relations between air quality and earth environment, etc. Previous studies in this domain would be generally divided into two groups: 
1) Time series analysis: It was often used to describe some basic features of air quality data [4]. For examples, Schwartz [5] found that there are strong correlations between air pollution levels and daily mortality in London. Kim [6] proposed a generalized linear model based on the time series data of ozone in Southern California, the model can effectively capture the seasonal non-stationary in ordinary time series. The relation between AQI and social-economical factors was also studied in [7], they analyzed the AQI of 31 provincial cities of China, and found that the value of AQI is positively correlated with the economic level and population level. $\mathrm{Li}$ [8] tried to infer the urban air condition from perspective of time series, they focused on $\mathrm{PM}_{2.5}$ based urban air quality, and introduced two kinds of time-series methods for real-time and fine-grained air quality prediction. They also proposed a model to show that the spatial scaling rules of population, roads and socioeconomic interactions are in a consistent framework [9]. Xu [10] presented a data analysis framework to uncover the impact of urban traffic on estimating air quality in different locations within a metropolitan area. They estimated the commuter's exposure to ambient $\mathrm{PM}_{2.5}$ by using the mobile phone data, the environmental justice in $\mathrm{PM}_{2.5}$ exposure was investigated by comparing the exposure with housing price [11].

2) Complex networks: It is an active area of studying the non-trivial topological features, and relations within the multi-agent systems [12-14]. It could be also used to study the features and evolution of the time series data [15-20]. Representative works, such as, Fan [21] studied the PM 2.5 time series data by networks. The phase spaces are denoted as nodes, and edges are assigned to nodes with higher correlation coefficients. They analyzed the relations between the criteria of correlation coefficients and the topological quantities, the similarities of different cities' air quality. Carnevale [22] use neural network to find the source of air pollutants, and found that the source of PM 10 is the easiest and most accurate to be located. Zhang [23] studied the correlation and scaling behaviors of PM 2.5 time series of different cities of China, and found that the probability distribution of the correlations has two peaks, the weighted degree distributions of networks with different kinds of correlations are also discussed. Du [24] established the correlation network by using the AQI datasets of 35 major cities, they found that the abrupt phase transition usually occurs between three to six weeks ahead of the peak or valley point of the evolution of the AQIs mean in highly polluted region. Zhang [25] studied the AQI datasets of Beijing, they transformed the AQI time series to symbol sequence, and studied the different patterns from the network perspective. Wei [26] studied the criticality evaluation of air quality standards by the network approach. the network was constructed by the relations of each standard, the critical standards are identified by measuring the centrality of nodes in the network.

Time series analysis and complex networks are two useful metrics for carrying out the quantitative analysis of the air quality data. However, most of the previous works did not consider different cities as a whole system (other than the fluctuations, there should be interactions and correlations), and the geographical factors are not taken into account when analyzing the temporal characteristic of air quality. Therefore, we 
dedicate to study both the air quality patterns and the correlations of different cities with more complete datasets as we can [27], that is, the AQI series data of totally 363 cities from Jan. 2015 to Mar. 2019.

Through calculating the Pearson Correlation Coefficients [28] of AQI series data between each pair of cities, we construct the AQNC by using the PMFG algorithm [2931]. The probability distribution of the geographical distances of cities which have direct links in AQNC shows that the air pollution has a strong correlation within $100 \mathrm{~km}$ and this correlation would become weak as the distance increases. Eight communities are detected in AQNC based on the edges centrality algorithm [32], the detection results are reasonable both for the large modularity and the geographical distribution. The air quality patterns of each community are studied by considering the long-term memory effects and geographical environments. To uncover the characteristics of the motifs of AQI series data, we use the visibility graph to explore the evolutionary patterns of motifs.

The rest of the paper is organized as follows. In Section 2, the AQNC is constructed by PMFG, the basic properties of AQI and the correlations of air quality of different cities also studied. In Section 3, the community structure of AQNC is detected, and the patterns of different communities are analyzed by taking into account the regional average Hurst exponents, average Hurst exponents of each city, etc. Section 4 shows the results of AQI evolutionary pattern using the visibility graph method. Conclusions and discussions are made in Section 5.

\section{Construction of AQNC and Correlation of AQI of different cities}

The comprehensive evaluation of AQI is based on the pollution index of $\mathrm{SO}_{2}, \mathrm{NO}_{2}$, $C O, P M_{2.5}, P M_{10}$, and $O_{3}$ [33]. We collected and processed the daily AQI time series data of 363 cities of China from Jan. 2015 to Mar. 2019 [27]. The AQI time series data is transformed to AQNC by employing the well-known PMFG method. Based on the correlation of AQI of different cities, the geographical distance on the air pollution diffusion has been investigated.

\subsection{The construction of $A Q N C$ and some basic properties of $A Q I$}

The air quality displays seasonal cycles, thus the datasets of AQI have strong seasonal effect. There are different methods of removing this effect, such as by subtracting the mean seasonal cycle and dividing by the seasonal standard deviation of each grid point time series $[34,35]$ or the curve-fitting method [36]. The time length of our AQI datasets

are around 4 years, the mean seasonal cycle would have large fluctuations, therefore we decide to use the curve-fitting method to remove the effect of seasonality, the calculation processes are as follows:

1) Fit the AQI time series data of each year by using the polynomial function with 5 parameters, so as to get the general trend of the datasets. 


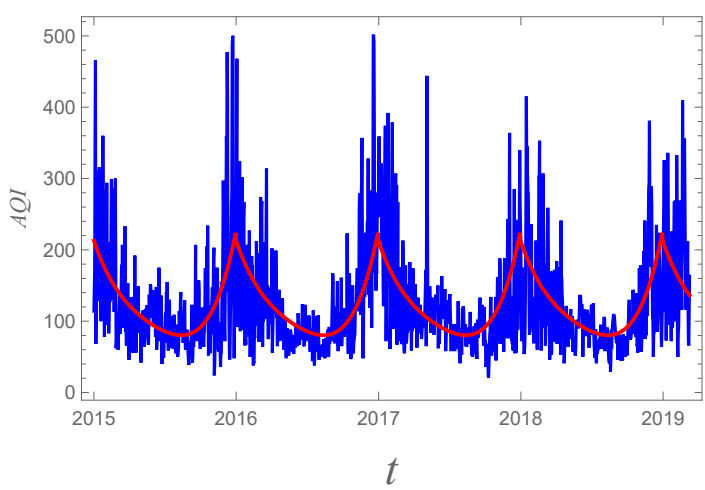

(a) The $A Q I$ and trend curve of Akesu.

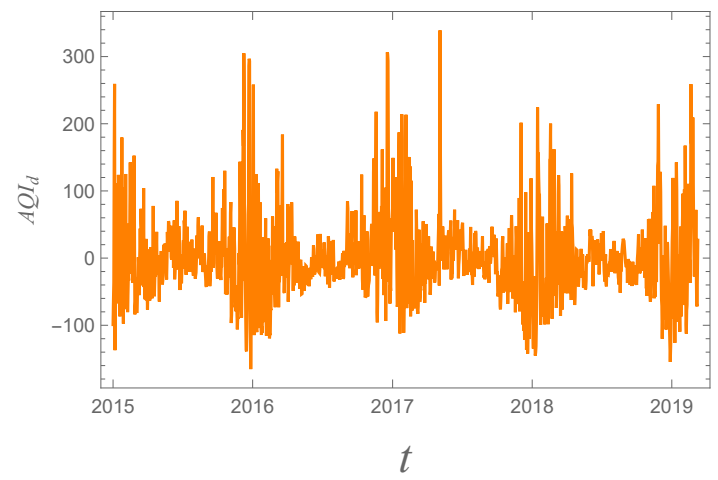

(b) Time evolution of $A Q I_{d}$ of Akesu.

Figure 1. (Color online) (a) The AQI time series of Akesu (blue curve), the red line is the fitting curve by employing the polynomial function. (b) The time series of the detrended datasets of AQI (denoted as $\mathrm{AQI}_{\mathrm{d}}$ ).

2) The detrended datasets (denoted as $\mathrm{AQI}_{\mathrm{d}}$ ) are obtained by subtracting the trenddatasets from the original datasets.

One example of this detrended process is shown in Fig. 1 for the city of Akesu. The blue and red cures on Fig. 1(a) represent the evolution of the original datasets and fitting trend datasets of AQI, respectively. The orange cure on Fig. 1(b) corresponds to the evolution of the detrended datasets of AQI (denoted as $\mathrm{AQI}_{\mathrm{d}}$ ). We have tested the seasonality trends of the $\mathrm{AQI}_{\mathrm{d}}$ by employing the Fourier transform method [37], and found that the periodogram has no obvious spike. We also calculate the cycle lengths of the top 10 peaks, all of them are not close to one year. Therefore, we would conclude that the seasonality trends of the detrended AQI data $\left(\mathrm{AQI}_{\mathrm{d}}\right)$ are removed.

The Pearson Correlation Coefficient is introduced to calculate the correlations of the detrended AQI time series data $\mathrm{AQI}_{\mathrm{d}}$ of $N=363$ cities. The $C_{i j}^{t, \delta}$ between cities $i$ and $j$ during the time period $\{t-\delta / 2, t+\delta / 2\}$ is defined as

$$
C_{i j}^{t, \delta}=\frac{\left\langle I_{i}^{t} I_{j}^{t}\right\rangle-\left\langle I_{i}^{t}\right\rangle\left\langle I_{j}^{t}\right\rangle}{\sqrt{\left[\left\langle I_{i}^{t^{2}}\right\rangle-\left\langle I_{i}^{t}\right\rangle^{2}\right]\left[\left\langle I_{j}^{t^{2}}\right\rangle-\left\langle I_{j}^{t}\right\rangle^{2}\right]}}
$$

where $\delta$ is the estimation interval, and $\langle\ldots\rangle$ is the sample mean over the detrended AQI series vector $\left\{I_{i}^{t}\right\}$ of city $i$. In the process of calculating the correlations between two cities' detrended AQI series, we shift the time series backwards and forwards from 1 day to 30 days. Then we calculate the correlation coefficients for the overlap time range of the detrended AQI time series of the two cities, and the largest value is assigned to the Pearson correlation coefficient between the two cities. After we calculate the $N \times N$ correlation coefficients $C_{i j}$ of each city $i$ and city $j$, we get the air quality correlation matrix.

The $N \times N$ correlation matrix is transformed to network by using the PMFG method [31], which is useful and effective to convert the time series data to the complex network, by showing the correlations between different time series datasets. The Pearson 


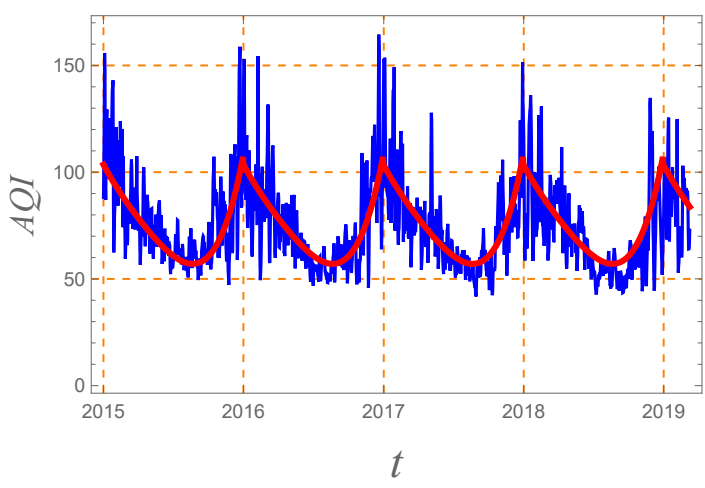

(a) Time evolution of $\langle A Q I\rangle$.

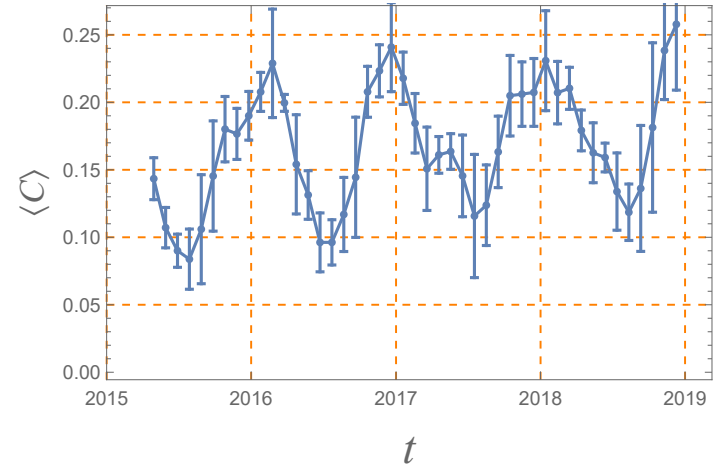

(b) Time evolution of $\langle C\rangle$.

Figure 2. (Color online) (a) The time evolution of $\langle A Q I\rangle$ (average AQI of 363 cities of China (blue line), the red line just displays the general trend of the evolution of $\langle A Q I\rangle$ to guide the eyes.). (b) The time evolution of $\langle C\rangle$ (average correlation coefficients $C$ between all cities with different correlation length $\delta$ ). The error bars represent the standard deviation of $\langle C\rangle$ for different correlation lengths $\delta$ ranging from 30 days to 180 days.

correlation coefficients $C_{i j}$ are ranked from the largest one to the smallest one. Then $3(N-2)$ edges are added between cities according to the correlation coefficients rank list (from the largest one), and the new edge adding process should keep the network as a planar graph.

The main advantage of this method is that, at the very beginning, edges are added between cities with larger Pearson correlation coefficients, after many edges are added, the overall picture of the network is almost clear. But there are still a few cities that are not connected to the network, and if new edges are added without the condition of keeping the network as a planar graph, there should be huge number of new edges to be added so as to connect all cities. So with this method, all nodes could be connected to the network with less edges, which makes the correlations between cities clean and clear. Moreover, the minimum spanning tree is more convenient and efficient to show the correlations between different cities. For example, the community structures of networks could be detected with better accuracy.

We have checked the Pearson correlation coefficients distribution of city-pairs with links in the network, normally their Pearson correlation coefficients are larger. This means that the network links in AQNC could reflect the overall picture of the correlations between cities.

The average correlation coefficients $\langle C\rangle$ of each pair of cities are calculated for different correlation length $\delta$ ranging from 30 days to 180 days (in order to reduce the fluctuations of only one constant correlation length). The evolutions of $\langle C\rangle$ and the average AQI of all cities are shown in Fig. 2. When $\langle A Q I\rangle$ is larger, $\langle C\rangle$ also becomes larger. While the peaks of the curve of $\langle C\rangle$ slightly shift to the left compared to that of the curve of $\langle A Q I\rangle$. It means that after $\langle C\rangle$ reaches the largest value, the air quality would become worse then. 


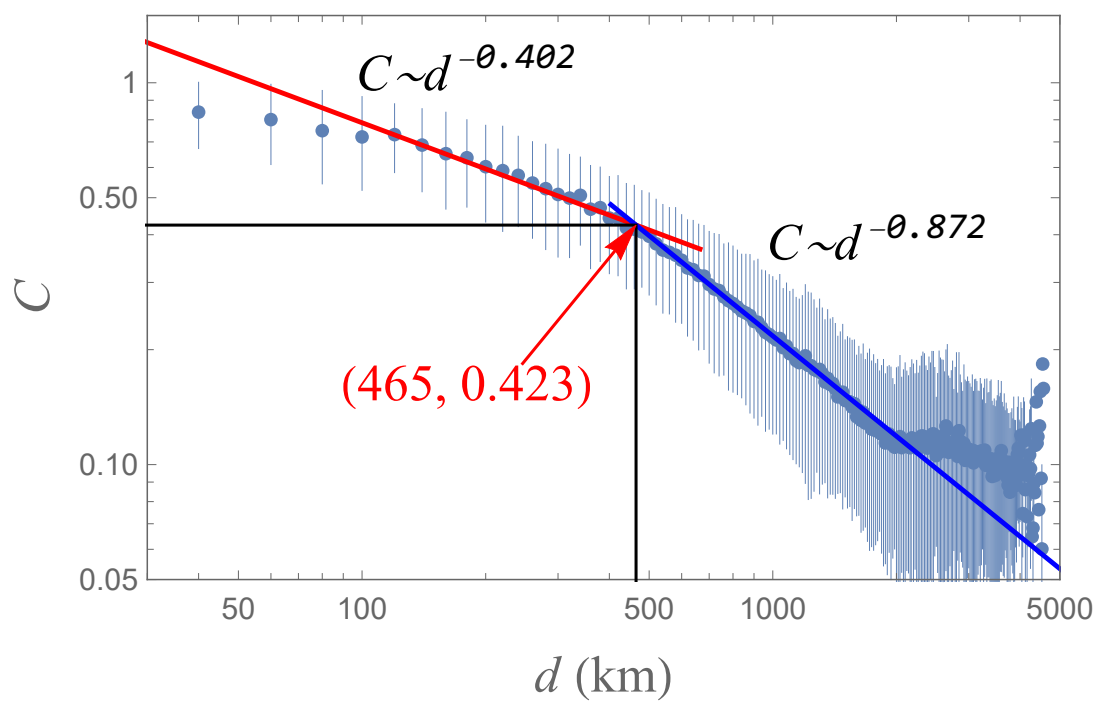

Figure 3. (Color online) The relationship between the geographical distances $d$ for each pair of cities and their corresponding Pearson correlation coefficients $C$ in double$\log$ scale. The red and blue curves are the power law fittings of the two regimes, the turning pint is $(465,0.423)$. The correlation coefficients are averaged over each bin of $10 \mathrm{~km}$, the error bars represent the standard deviations of $C$ over each bin.

\subsection{Air pollution diffusion distance}

Wind is an important factor that affects the diffusion of air pollutions [23]. Normally the closer the geographical distance of two cities, the stronger the correlation of their AQI would be. Therefore, we investigate the relationship between the Pearson correlation coefficients of any two cities and their geographical distances,and plot them in the double-log scale (see Fig. 3). One could observe that there are two regimes of the relationship, i.e., double power laws with two different slopes. The turning point is $(d=$ $465 \mathrm{~km}, C=0.423)$. It means that, when $d>465 \mathrm{~km}$, the correlations between cities would become weaker.

Boers [38] studied the global rainfall teleconnections by calculating the probability distribution of distances of links between different places within the network. They found a critical distance of $2500 \mathrm{~km}$, within the critical distance, the rainfall shows a regional weather system, when the distance is larger than $2500 \mathrm{~km}$, the rainfall teleconnections are the global-scale ones. In this realm, we both investigate the probability distribution of geographical distances of any two cities and of the connected cities in the AQNC, the results are shown in Fig. 4(a) and (b), respectively. The probability distribution of distances of any two cities follows $P(d) \sim e^{-0.00175 d} d^{1.546}$, the peak of the distribution is around $1000 \mathrm{~km}$. But the peak of the probability distribution of distances of links in AQNC is around $100 \mathrm{~km}$. There are two regimes of the distribution, the critical distance is around $100 \mathrm{~km}$. In the region of $(10,100) \mathrm{km}$, the probability distribution of distance follows $P(d) \sim d^{1.54}$, while in the region of $(100,1000) \mathrm{km}, P(d) \sim d^{-1.99}$. It means that most of the geographical distances of the connected cities in AQNC are around $100 \mathrm{~km}$. 


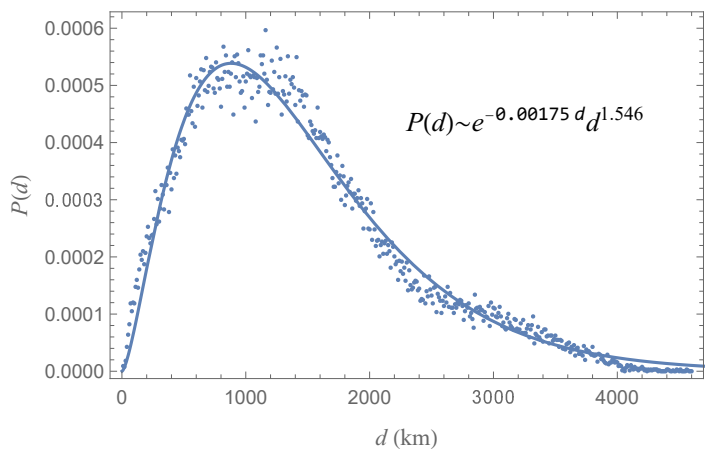

(a) Geographical distance distribution of any two cities among 363 cities.

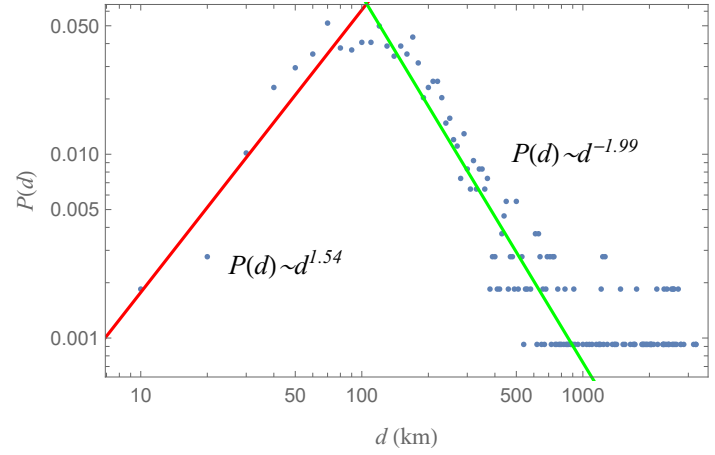

(b) Geographical distance distribution of the connected cities in AQNC.

Figure 4. (Color online) (a) The geographical distance distribution of any two cities among 363 cities. The peak of the distribution is around $1000 \mathrm{~km}$. (b) The geographical distance distribution of two cities that have direct links in the network. It is plotted in log-log scale, red line is the fitting cure in the range of $(10,100) \mathrm{km}$, while the green line is the fitting curve in the range of $(100,1000) \mathrm{km}$.

In China, $100 \mathrm{~km}$ is more or less the geographical distance between two nearby cities (cities that are in our AQI data list), the air qualities have strong correlations between them. Also, comparing Fig. 4(a) (geographical distance distribution of any two cities) and Fig. $4(\mathrm{~b})$, in the range of $[0,100] \mathrm{km}$, we observe that both of the two geographical distance distributions have the similar form with $P(d) \sim d^{1.54}$, this indicates that if the geographical distance of two cities is smaller than $100 \mathrm{~km}$, there would be a link between them in the network, so there are strong correlations between them.

Furthermore, the relationship between the average AQI (over all the periods) of a single city $\left\langle A Q I_{S}\right\rangle$ and its neighboring cities' average AQI $\left\langle A Q I_{N}\right\rangle$ have been investigated. Considering the relationship between Pearson correlation coefficients and geographical distances of two cities in Fig. 3, we assign $465 \mathrm{~km}$ as the neighboring influential range. Results are shown in Fig. 5(a), one can observe that the larger the average AQI of a city, the larger the average AQI of its neighboring cities. The probability distribution of the correlation coefficients between each city's $\mathrm{AQI}_{\mathrm{d}}$ and its neighboring cities' average AQI $\mathrm{d}_{\mathrm{d}}$ is shown in Fig. $5(\mathrm{~b})$, it is obvious that the peak of the distribution is around 0.75 , which shows very strong positive correlations. All these results demonstrate that the neighboring cities can have very similar AQI patterns.

\section{Communities of AQNC and their patterns}

In complex network, a community is a group of nodes which are relatively densely connected to each other within the group but sparsely connected to nodes in other groups of the network [39]. Detecting communities [40-42] can not only uncover the correlations between internal structures and functional behaviors of networks, but also have many practical applications in domains such as biology, sociology, economics and 


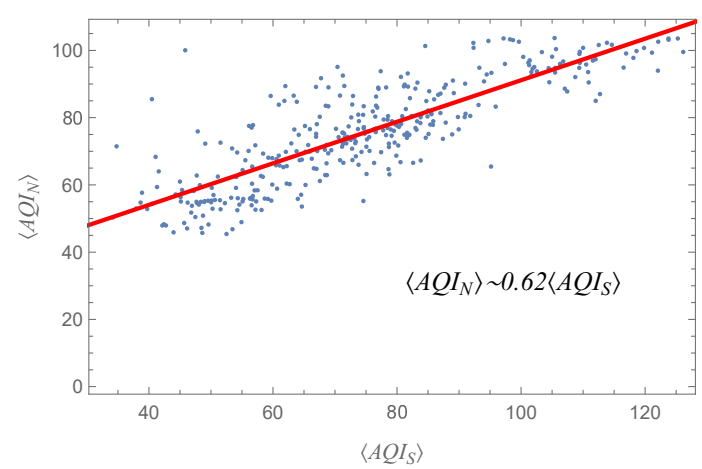

(a) Average AQI of a single city versus the average AQI of its neighboring cities within $465 \mathrm{~km}$.

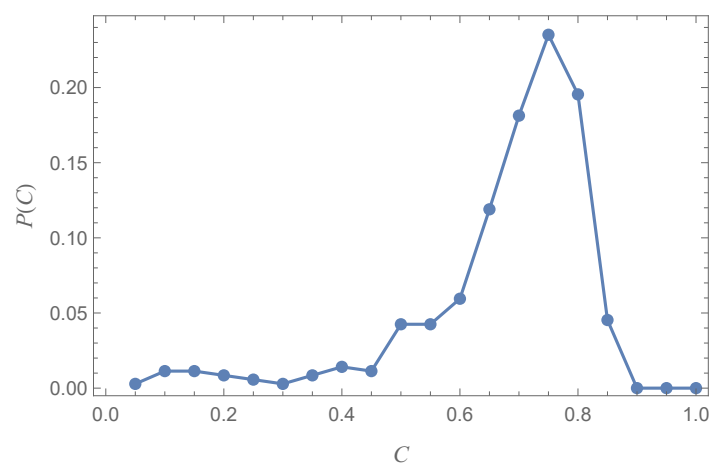

(b) Probability distribution of the correlation coefficients between each city's $\mathrm{AQI}_{\mathrm{d}}$ and its neighboring cities' average $\mathrm{AQI}_{\mathrm{d}}$.

Figure 5. (Color online) (a) The relationship between the average AQI of a single city $\left\langle A Q I_{S}\right\rangle$ and the average AQI of its neighboring cities $\left\langle A Q I_{N}\right\rangle$ within $465 \mathrm{~km}$. (b) The probability distribution of the correlation coefficients between each city's detrended AQI series data and its neighboring cities' average detrended AQI series data.

climate science, etc.

In our studies, the community means that, the AQI time series of cities within one community have the same pattern, i.e., the evolution features of AQI are similar to each other, so they have strong correlations. The community structure of AQNC is detected based on the edge centrality algorithm [32], the result is compared with the geographical location of cities [43]. The Hurst exponents of a city's AQI series data and a community's $\langle A Q I\rangle$ series data are calculated, respectively. By taking into account the monsoonal distribution, precipitation distribution and other geographical climate factors, we analyze the different patterns of AQI time series belonging to different communities.

\subsection{Results of community detection}

As shown in Fig. 6(a), eight communities of AQNC have been detected, the value of modularity is 0.807 , it means that the community detection result is accurate and reliable, i.e., the AQNC has obvious community structure. From Fig. 6(b), one could find that the cities belonging to the same community almost locate in the same region, this indicates that the air quality of a city is largely influenced by its geographical location, i.e., the similar pattern of air quality is mostly due to the similar geographical environment. The locations of the eight communities and their geographical features are as follows [44-46]:

1) Southeast China: Area with extreme high precipitation, the cities locate on the monsoonal path.

2) East China: Plain area with medium precipitation, close to the East China Sea.

3) Southwest highland of China: Highland area with rough terrain, medium precipitation and low wind. 


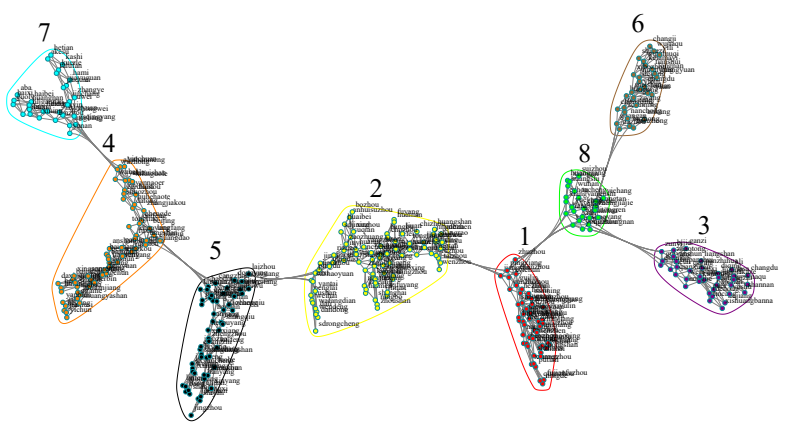

(a) Community struture of AQNC.

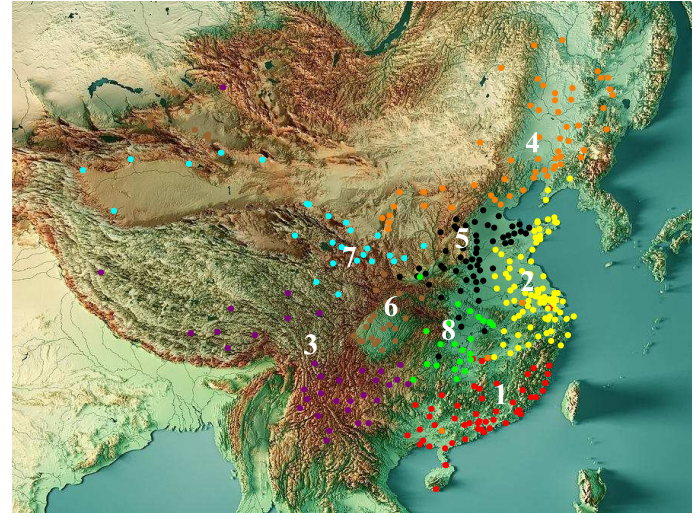

(b) Geographical locations of cities of different communities.

Figure 6. (Color online) (a) The community detection result of AQNC. It contains 8 communities and the cities of the same community are denoted by the same color. (b) The geographical locations of cities of different communities on the Chinese map, the colors of cities are the same as shown in (a). The background of figure is the landforms map of China [46].

4) Northeast China: Plain area with low precipitation and high wind.

5) Central-north China: Plain area with medium precipitation and low wind.

6) Basin of China: Basin area with medium precipitation and low wind.

7) Gobi desert of China: Gobi desert area with rough terrain, low precipitation and high wind.

8) Central-south China: Plain area with high precipitation and medium wind.

\subsection{AQI pattern analysis by Hurst exponent and environmental factors}

The Hurst exponent is employed as a measure of the long-term memory of time series. It relates to the autocorrelations of the time series, and the rate at which these decrease as the lag between pairs of values increases [47]. We calculate the Hurst exponent by using the rescaled range analysis (R/S analysis) [48], for a time series of length $n$, $X=X_{1}, X_{2}, \ldots, X_{n}$, the rescaled range is calculated as follows:

$$
\begin{aligned}
& m=\frac{1}{n} \sum_{i=1}^{n} X_{i}, \\
& Y_{t}=X_{t}-m \quad \text { for } \quad t=1,2, \ldots, n, \\
& Z_{t}=\sum_{i=1}^{t} Y_{i} \quad \text { for } \quad t=1,2, \ldots, n, \\
& R(n)=\max \left(Z_{t}\right)-\min \left(Z_{t}\right) \quad \text { for } t=1,2, \ldots, n, \\
& S(n)=\sqrt{\frac{1}{n} \sum_{i=1}^{n}\left(X_{i}-m\right)^{2}}
\end{aligned}
$$


where $m$ is the mean value of the series data, $Y_{t}$ is the mean-adjusted series, $Z_{t}$ is the cumulative deviation series, and $R(n)$ represents the range of deviation, $S(n)$ is the standard deviation (normalization factor). Using the above formulas, we can calculate the rescaled range $R / S(n)=R(n) / S(n)$, averaging all the partial time series by length $n$, and obtain $R / S(n) \sim n^{H}$, in which $H$ is the Hurst exponent. In the calculation process for Hurst exponent, we found that the R/S line becomes not smooth when $n>365$, so we set one year as the upper bound length of long-term memory. Values of Hurst exponents of the AQI time series of all 363 cities are shown in Fig. 8(a), and the value of the Hurst exponent $H_{A}$ of the average AQI time series of all cities is 0.955. These results indicate that the AQI time series have the strong long-term memory effects, regardless of the climate patterns or geographical locations of the cities.

We average the AQI time series of cities within the same community, and calculate each community's Hurst exponent denoted as $H_{C}$ (see Table. 1 and Fig. 8(b)). The community's Hurst exponent $H_{C}$ was compared with the average Hurst exponent of all single cities within the community (denoted as $\left\langle H_{S}\right\rangle$ ) in Fig. 7. The $H_{C}$ is larger than $\left\langle H_{S}\right\rangle$ for each community, since the time series of average AQI of a community has smaller fluctuations. $H_{C}$ and $\left\langle H_{S}\right\rangle$ are linearly correlated except community 4 (Orange color point) and community 7 (Cyan color point) that exhibits an abnormal feature (see Fig. 7). The Hurst exponents of different communities posses their own features, since for each community, it has its own geographical aggregation effect. The datasets of the precipitation, monsoon, and wind energy data of different areas are obtained from the previous studies and literatures [44-46]. Thus for example we roughly divide the precipitation into four levels according to their values, and got the levels of low, medium, high and extreme high. The geographical characteristics of each community, including the precipitation, monsoon, wind, regional geomorphic feature and average Pearson correlation coefficient $\left\langle C_{c}\right\rangle$, are presented in Table. 1.

From the community analysis of AQNC, we can discuss the results as follows: 1) The values of the $\left\langle H_{S}\right\rangle$ and $H_{C}$ are relatively large in the basin area, due to the very low wind/air exchanges, which implies that the AQI time series of the basin area has strong long-term memory. 2) The wind and precipitation are negatively correlated with the Hurst exponents of single cities, as well as communities. Since the wind and precipitation could influence very large area, so as to break the air pollution accumulative effect [49], and lessen the Hurst exponents. 3) The highland and gobi desert areas have rough terrain which can lead to low correlation between cities (small $\left\langle C_{c}\right\rangle$ ), and large $H_{C}$, such as communities 3 and 7 . The community 7 have high wind that leads to small $\left\langle H_{S}\right\rangle$, but rough terrain reduces the air flow and leads to large $H_{C}$. 4) The wind (local area) could reduce the value of the Hurst exponent of single cities, but reduce the value of communities $\left(H_{C}\right)$ slightly. Since it can only influence the AQI change in smaller area (single city), and it is not the same as monsoon which can influence large area (the community area), such as community 4. 


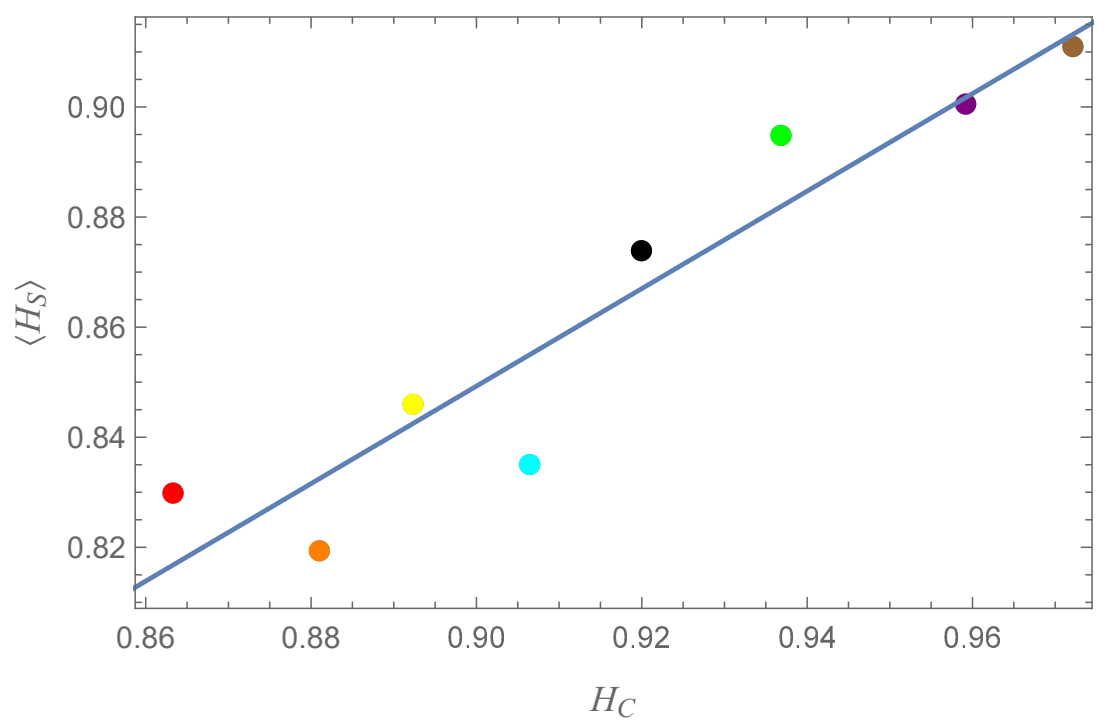

Figure 7. (Color online) The relation of a community's Hurst exponent $H_{C}$ versus the average Hurst exponent of all single cities within the community $\left\langle H_{S}\right\rangle$. As can be seen, they are linearly correlated, except community 4 with orange color and community 7 with cyan color.

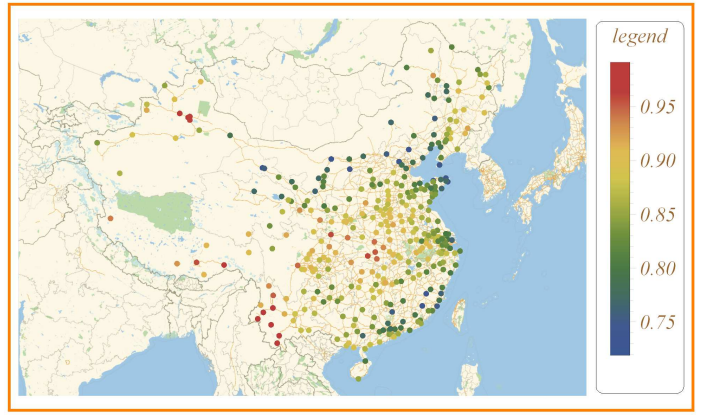

(a) Hurst exponents of 363 cities.

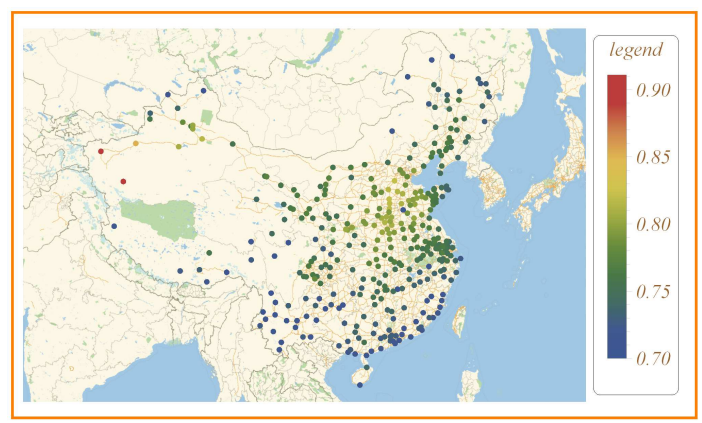

(c) The average AQI of 363 cities.

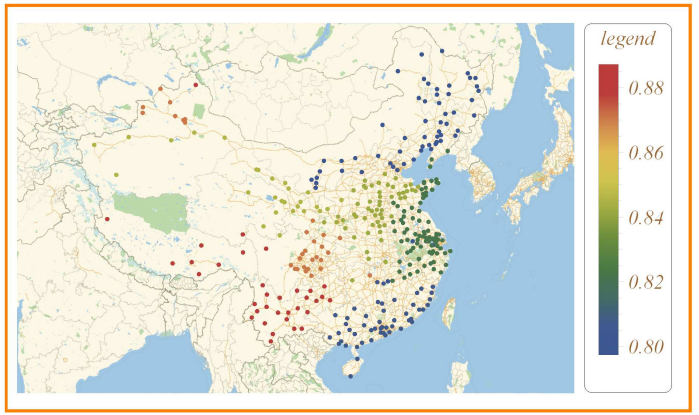

(b) Hurst exponents of 8 communities.

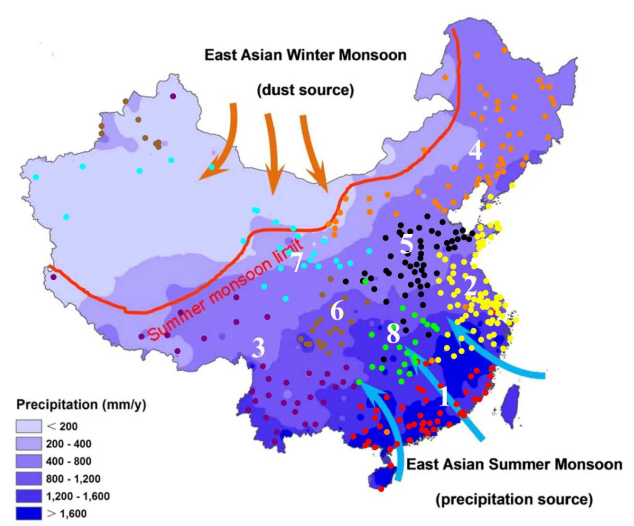

(d) Community versus geographical environment.

Figure 8. (Color online) The heat map of the Hurst exponents of (a) 363 cities and (b) 8 communities. (c) The heat map of the average AQI of the 363 cities. (d) The geographical distribution of precipitation and monsoon versus communities (the background map is cited from [45]). 
Table 1. Basic quantities and features of the 8 communities. $\langle A Q I\rangle$ is the average AQI of a community through the whole period. $H_{C}$ is the Hurst exponent of a community. $\left\langle H_{S}\right\rangle$ is the average Hurst exponent of all single cities within the community. $\left\langle C_{c}\right\rangle$ is the average Pearson correlation coefficient among cities of the same community with distance being less than $465 \mathrm{~km}$.

\begin{tabular}{|c|c|c|c|c|c|c|c|}
\hline $\begin{array}{l}\text { Community } \\
\text { label }\end{array}$ & Color & $\langle A Q I\rangle$ & $H_{C}$ & $\left\langle H_{S}\right\rangle$ & $\left\langle C_{c}\right\rangle$ & Precipitation & Geographical features \\
\hline 1 & Red & 54.46 & 0.863 & 0.83 & 0.630 & Extreme High & $\begin{array}{l}\text { Close to ocean, in } \\
\text { monsoon path }\end{array}$ \\
\hline 2 & Yellow & 74.99 & 0.892 & 0.846 & 0.576 & Medium & $\begin{array}{l}\text { Close to ocean, not in } \\
\text { monsoon path }\end{array}$ \\
\hline 3 & Purple & 49.84 & 0.959 & 0.901 & 0.424 & Medium & Highland, low wind \\
\hline 4 & Orange & 73.04 & 0.881 & 0.819 & 0.569 & Low & Plain, high wind \\
\hline 5 & Black & 103.37 & 0.92 & 0.874 & 0.558 & Medium & Plain, low wind \\
\hline 6 & Brown & 76.09 & 0.972 & 0.911 & 0.625 & Low & Basin, low wind \\
\hline 7 & Cyan & 88.46 & 0.906 & 0.835 & 0.481 & Low & Gobi desert, high wind \\
\hline 8 & Green & 75.25 & 0.937 & 0.895 & 0.659 & High & Plain, medium wind \\
\hline
\end{tabular}

\section{Motifs of AQI time series by the visibility graph}

The visibility graph method was used to analyze evolution features, especially the motifs of AQI time series. Some standard motifs were recognized by comparing the original time series with the shuffled ones. We further studied the stability of these motifs of different communities by checking their long-term memory effects.

\subsection{The process and properties of visibility graph}

The visibility graph was proposed by Lacasa [15] and used to transform the time series to graphs, then these graphs are regarded as vertices to construct a new network [50]. Here, we choose five timing points from $t$ to $t+4$ and set every timing point as one vertex in the network, if two elements (e.g., with height being the value of AQI) at different timing points are visible, they will be connected in the network (see Fig. 9(a)). So we considered each time window with length 5 , and set every timing point as a vertex. An edge is added if two vertices (with the height of AQI value) could see each other, for which the direction is from the early timing vertex to the subsequent one. Please find the schematic diagram in Fig. 9. Fig. 9(c) is the corresponding adjacency matrix of the graph in Fig. 9(b). The values of elements on the red line are 1 since they reflect the adjacent timing points. The values of elements below the diagonals are 0 . Thus we can represent the visibility graph by the elements above the red line. According to the previous results [51], for 5 timing points, the connection patterns in the visibility graph can be divided into 25 kinds.

We denote the network composed of 5 timing vertices as a mode $g_{t}$, and the next shifted (only one step) 5 timing vertices as $g_{t+1}$, and so on. So the time series of AQI can be denoted as $g_{t}, g_{t+1}, g_{t+2}, \ldots, g_{t+n}$. For the next step, we add an edge for 
any two consecutive mode $g_{t}, g_{t+1}$, and the same mode of visibility graph as one point, then the network is constituted by 25 visibility graphs (most visibility graphs can't be generated), and the mutual transformation can be obtained, which is named as the visibility network. The network is shown in Fig. 10, which could reflect the patterns and rules of AQI evolution. Each node corresponds to a visibility graph. The digit next to the node is the name of the visibility graph, in the way that, if we transform that name-digit to the binary number with nine-digits ( 0 or 1 ), then these nine-digits ( 0 or 1) just correspond to the nine numbers at the top right-hand corner of the adjacent matrix of visibility graph. So the name-digit could reflect the very basic information of the visibility graph. The larger points imply the patterns appear in AQI series much more frequently, and the thicker line means the two patterns have higher probability of transforming from one to another. We calculate the top 8 nodes (visibility graphs) with large frequencies of all communities (see Table. 2).

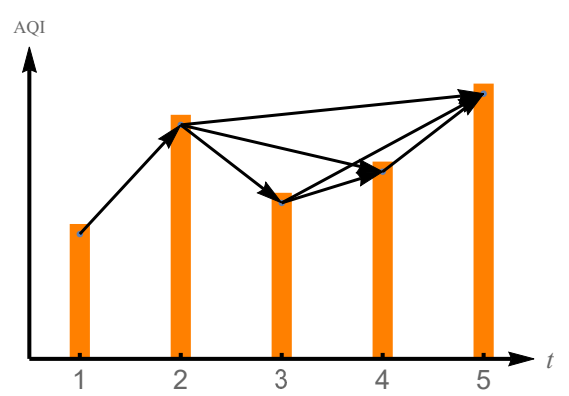

(a) Time series.

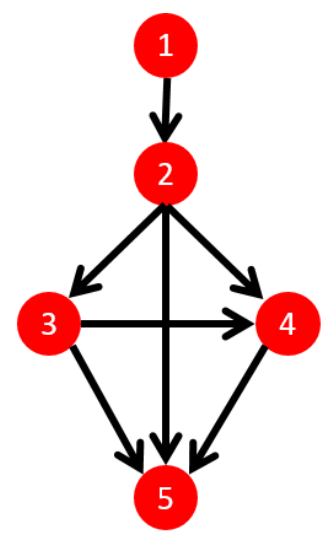

(b) Visibility graph mode.

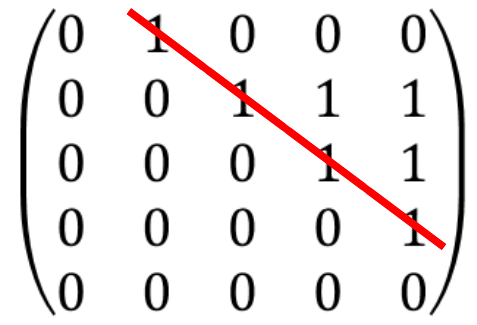

(c) Adjacent matrix.

Figure 9. (Color online) The process of transforming the time series to visibility graph and its corresponding adjacent matrix.

\subsection{Characteristics of Motifs of the AQI time series}

To investigate the characteristics of motifs of AQI time series, we shuffle the AQI series and construct some new visibility networks, then we compare the frequency of each visibility graph between the two visibility networks. If the degree of an original visibility network is larger than that of the shuffled visibility network, this visibility graph could be called the "motif pattern". The different visibility graphs are ranked by the distinction between the original time series and the shuffled one. The top five motifs and corresponding time series of eight communities are shown in Tab. 3. The motifs are not the same with the top frequency visibility graphs (Table. 2), and the motifs 1 and 2 of all communities are the same.

Then we calculate the Hurst exponents of motifs in eight communities. We implemented the metric [50] of calculating the Hurst exponent of discrete time series 


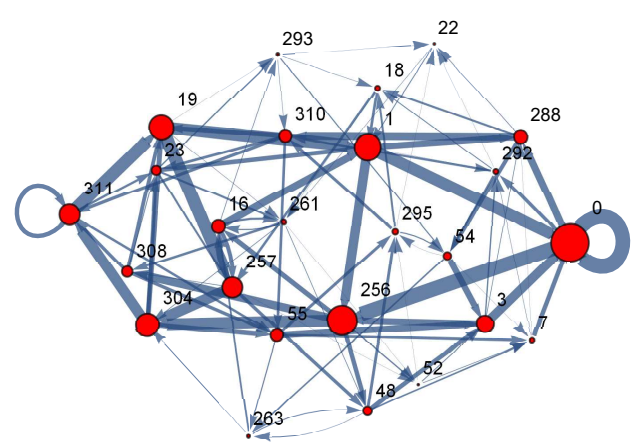

(a) Southeast China.

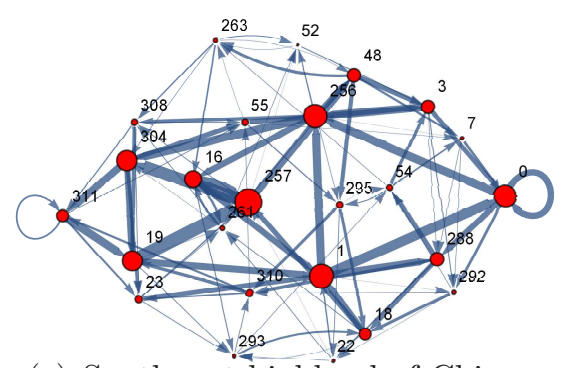

(c) Southwest highland of China.

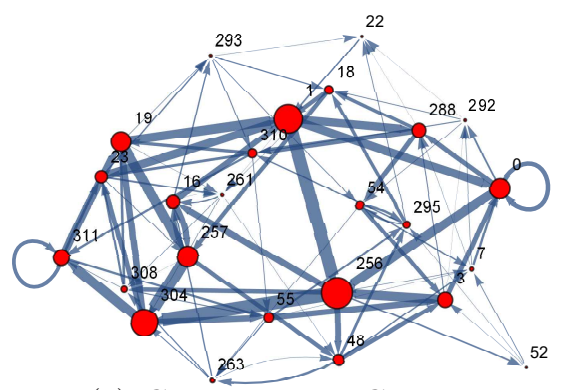

(e) Central-north China.

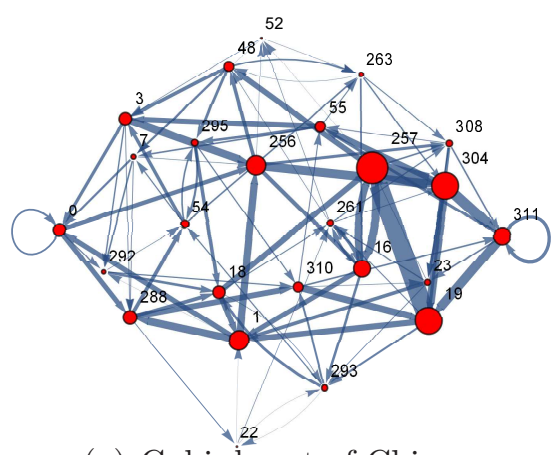

(g) Gobi desert of China.

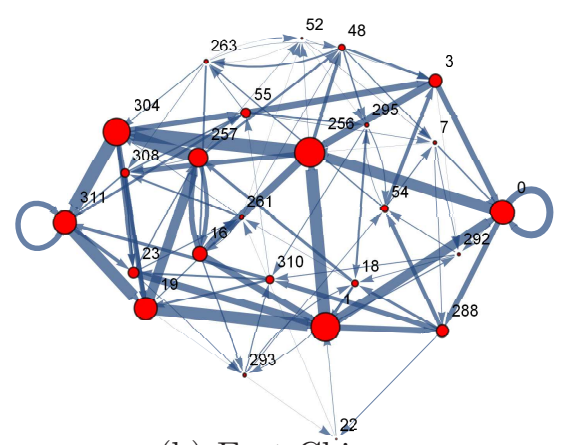

(b) East China.

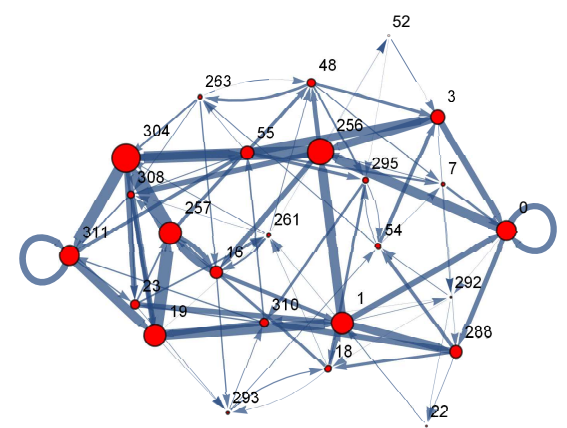

(d) Northeast China.

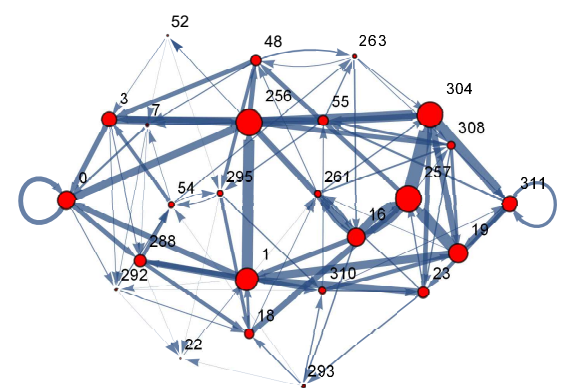

(f) Basin of China.

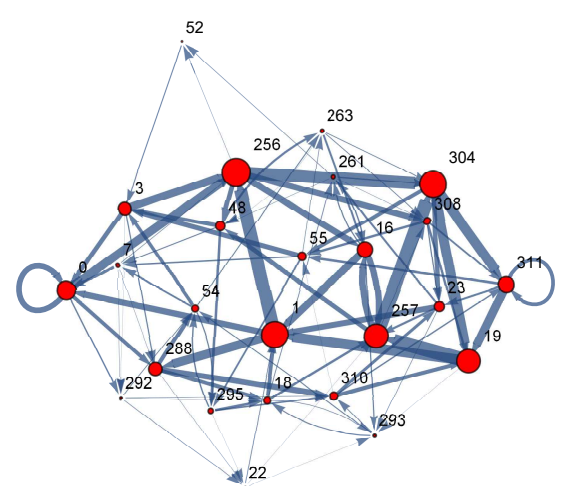

(h) Central-south China.

Figure 10. (Color online) The transforming patterns of the visibility graphs of eight communities, the thickness of the line is proportional to the frequency of that transformation, and the size of the vertex is proportional to the frequency of the visibility graph in the AQI time series. The labels of the visibility graphs are named in the way that, if we transform that label to the binary number with nine-digits (0 or 1$)$, then these nine-digits ( 0 or 1$)$ just correspond to the nine numbers at the top right-hand corner of the adjacent matrix of visibility graph. 
with different interval as follows: The top three motifs are chosen to calculate the Hurst exponents of AQI series. Firstly, we denote the time of the chosen motif as $\omega_{k}$, where $k=1,2, \ldots, M$ means the motif is the $k$-th appeared one in the time series. Then we set

$$
\Omega^{j}=\left(\omega_{j+1}-\omega_{j}, \omega_{j+2}-\omega_{j+1}, \ldots, \omega_{j+n}-\omega_{j+n-1}\right),
$$

where $j=1,2, . ., M-n$, then

$$
\begin{aligned}
\Phi^{j}(i) & =\sum_{w=1}^{i}\left[\Omega^{j}(w)-\left\langle\Omega^{j}\right\rangle\right] \\
& =\omega_{j+1}-\omega_{j}-\frac{i}{n}\left(\omega_{j+n}-\omega_{j}\right) \quad(i=1,2, \ldots, n .) .
\end{aligned}
$$

The Hurst exponent can be calculated as

$R / S(n)=\frac{1}{M-n} \sum_{j-1}^{M-n} \frac{\max \left[\Phi^{j}(1), \Phi^{j}(2), \ldots, \Phi^{j}(n)\right]-\min \left[\Phi^{j}(1), \Phi^{j}(2), \ldots, \Phi^{j}(n)\right]}{\operatorname{std}\left(\Omega^{j}\right)}$.

In the relation $R / S(n) \sim n^{H_{G}}, H_{G}$ is Hurst exponent of the visibility graph pattern. Since the fluctuations of AQI series of single city are large, we average the AQI series of all cities within one community, then we can obtain eight average AQI series. As is the same calculation process of the Hurst exponent, the R/S line becomes not smooth when $n>27$, thus we can calculate $H_{G}$ by setting $n$ with different values in the range of $(1,27)$. Otherwise the fourth and the subsequent motifs in each communities have low frequencies, their Hurst exponents could not be calculated, therefore we have only studied the top three motifs. The relation between $R / S$ and $n$ is presented in Fig. 11 and the $H_{G}$ exponents of all the communities are listed in Table. 4. Results show that most of the $H_{G}$ exponents are larger than 0.5 or approximately equal to 0.5 . Therefore, the

Table 2. The top 8 large frequency visibility graphs and corresponding time series of eight communities. The labels of the visibility graphs are the same as in Fig. 10.

Graph label

Graph label


Chinese cities' air quality pattern and correlation

Table 3. The top five motifs and corresponding time series of eight communities.

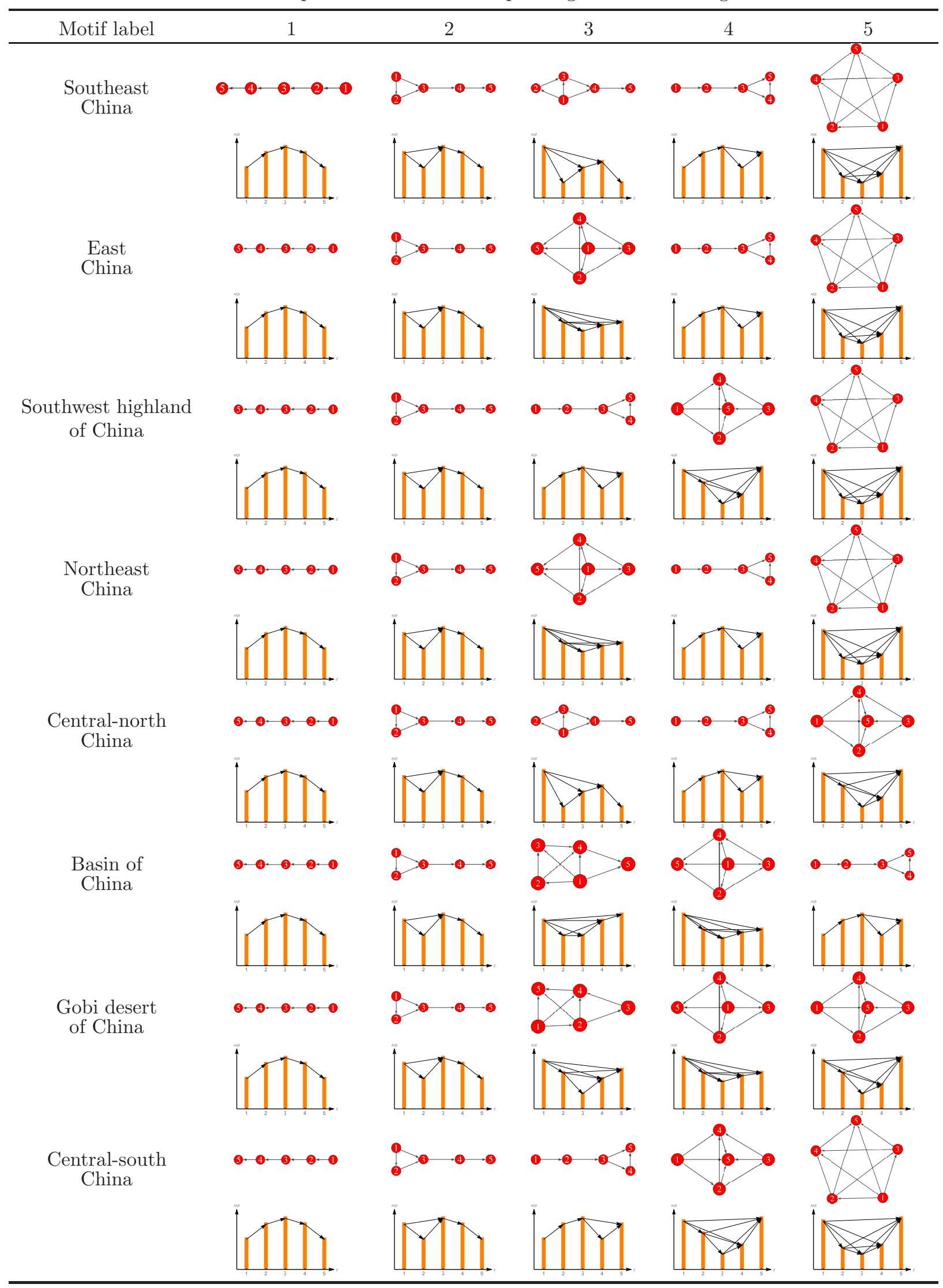




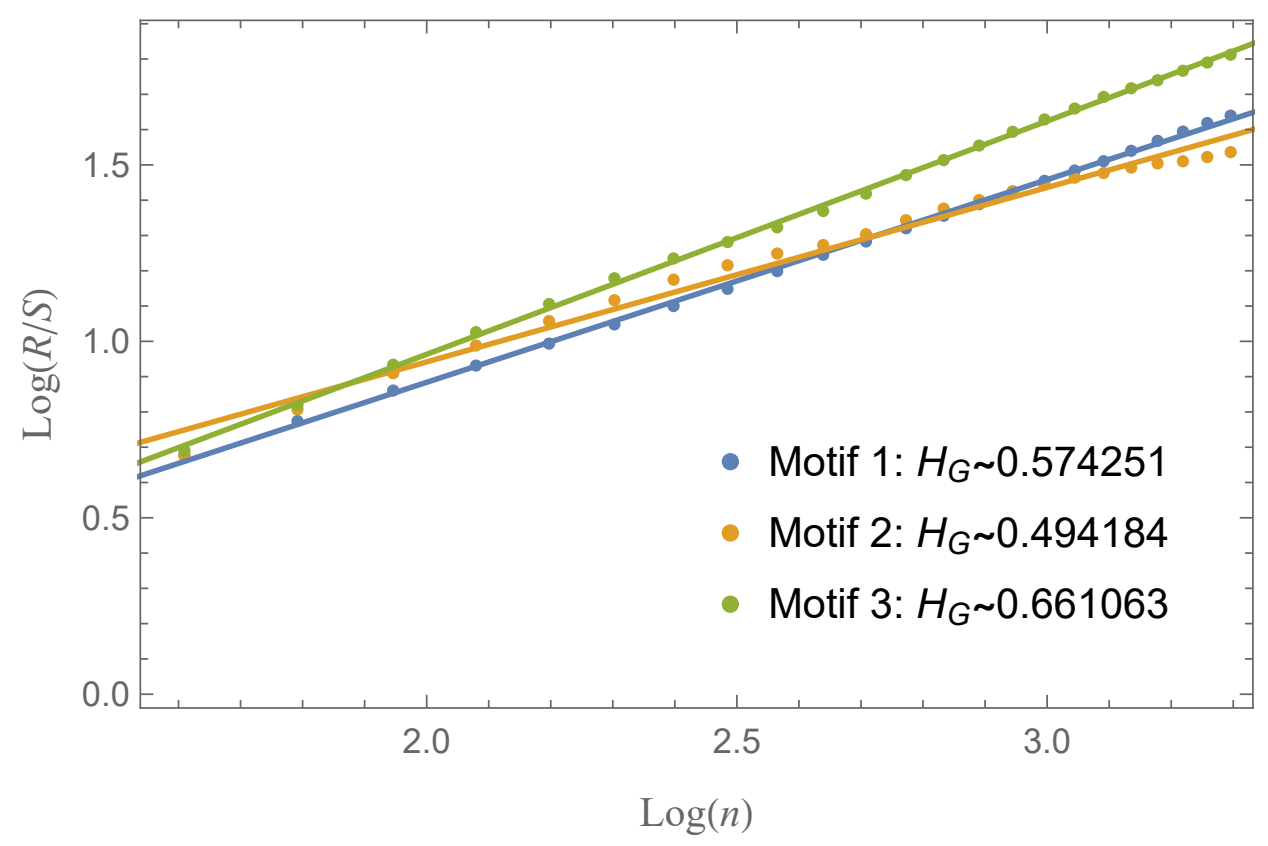

Figure 11. (Color online) The relation between $R / S$ and $n$ of motifs, it is plotted in $\log$-log scale. The different colors represent different motifs, and the dotted lines are the calculated results, the fitting results are the real lines.

Table 4. The $H_{G}$ exponent of top three motif in 8 average AQI series, and most $H_{G}$ exponents are larger than 0.5 or nearly equals to 0.5 .

\begin{tabular}{lcccccccc}
\hline Community label & 1 & 2 & 3 & 4 & 5 & 6 & 7 & 8 \\
\hline Motif 1 & 0.574 & 0.546 & 0.512 & 0.728 & 0.726 & 0.639 & 0.486 & 0.765 \\
Motif 2 & 0.494 & 0.623 & 0.624 & 0.631 & 0.619 & 0.663 & 0.609 & 0.503 \\
Motif 3 & 0.661 & 0.579 & 0.611 & 0.735 & 0.597 & 0.617 & 0.531 & 0.583 \\
\hline
\end{tabular}

evolutionary patterns have the long-term memory effect and the motifs always appear next if this motif have appear frequently.

\section{Conclusion}

We have analyzed the AQI time series from network perspective by using the PMFG method. The correlations of AQI between different cities have been calculated, it is found that there are strong correlations between cities within $100 \mathrm{~km}$. Eight communities of the AQNC have been found, and we observe that the cities in the same community almost distribute in the same region. We have also calculated the regional Hurst exponent and single city's Hurst exponents, respectively, and found that the AQI time series have strong long-term memory effect. Furthermore, we have used the precipitation, monsoon, and geographical environment to explain the pattern of AQI, regional Hurst exponent, and average Hurst exponent of single cities in every community. Lastly, we have transformed the AQI time series to visibility graphs, and got the motifs of eight 
communities. The Hurst exponents of motifs have been calculated, and results indicate that the evolutionary patterns of AQI in most communities are have long-term memory.

\section{Acknowledgements}

We gratefully acknowledge the fruitful discussions with Rongrong Xie, Longfeng Zhao, and Shengfeng Deng. This work was supported in part by National Natural Science Foundation of China (Grant No. 11505071, 11747135, 11905163, 61873104), the Programme of Introducing Talents of Discipline to Universities under Grant No. B08033 the Fundamental Research Funds for the Central Universities (Grant No.

KJ02072016-0170, CCNU, CCNU19QN029, CCNU19ZN012), the China Postdoctoral Science Foundation (Grant Nos. 3020501003).

\section{References}

[1] Kenneth W, Warner C F and Davis W 1998 Third Ediction, Eddison Wesley, USA 168-169

[2] Pope C A, Thun M J, Namboodiri M M, Dockery D W, Evans J S, Speizer F E and Heath C W 1995 Am J Respir Crit Care Med 151 669-674

[3] Fakinle B, Sonibare J, Okedere O, Jimoda L and Ayodele C 2016 Cogent Environmental Science 21208448

[4] Brockwell P J and Davis R A 1989 Technometrics 31 121-121

[5] Schwartz J and Marcus A 1990 American Journal of Epidemiology 131185

[6] Kim S E 2017 Environmental Modeling \& Assessment 22 175-182

[7] Xu L J, Zhou J X, Guo Y, Wu T M, Chen T T, Zhong Q J, Yuan D, Chen P Y and Ou C Q 2016 Air Quality Atmosphere \& Health 10 1-9

[8] Li R, Chen Y, Zhao X, Hu Y and Xiao W 2016 Big Data \& Information Analytics 1 171-183

[9] Li R, Dong L, Zhang J, Wang X, Wang W X, Di Z and Stanley H E 2017 Nature Communications 81841

[10] Xu Y, Li R, Jiang S, Zhang J and González M C 2017 Clearer skies in beijing-revealing the impacts of traffic on the modeling of air quality Report 17-05211 Transportation Research Board 96th Annual Meeting

[11] Xu Y, Jiang S, Li R, Zhang J, Zhao J, Abbar S and González M C 2019 Computers, Environment and Urban Systems $\mathbf{7 5}$ 12-21

[12] Erdős P and Rényi A 1960 Publ. Math. Inst. Hung. Acad. Sci 5 17-60

[13] Watts D J and Strogatz S H 1998 Nature 393440

[14] Barabási A L and Albert R 1999 Science 286 509-512

[15] Lacasa L, Luque B, Ballesteros F, Luque J and Nuno J C 2008 Proceedings of the National Academy of Sciences $1054972-4975$

[16] Zhang J and Small M 2006 Physical Review Letters 96238701

[17] Marwan N, Donges J F, Zou Y, Donner R V and Kurths J 2009 Physics Letters A 373 4246-4254

[18] Yang Y and Yang H 2008 Physica A: Statistical Mechanics and its Applications 387 1381-1386

[19] Bezsudnov I and Snarskii A 2014 Physica A: Statistical Mechanics and its Applications 414 53-60

[20] Gao Z K, Small M and Kurths J 2017 EPL (Europhysics Letters) 11650001

[21] Fan X, Wang L, Xu H, Li S and Tian L 2016 Environmental Science and Pollution Research 23 3621-3631

[22] Carnevale C, Finzi G, Pisoni E and Volta M 2009 Atmospheric Environment 43 4811-4821

[23] Zhang Y, Chen D, Fan J, Havlin S and Chen X 2018 EPL (Europhysics Letters) 12258003

[24] Du R, Li J, Dong G, Tian L, Qing T, Fang G and Dong Y 2019 Physica A: Statistical Mechanics and its Applications 123312 
[25] Zhang Y and Na S 2018 Sustainability 101073

[26] Wei Y, Chen L, Qi Y, Wang C, Li F, Wang H and Chen F 2019 Sustainability 113920

[27] http://www.tianqihoubao.com/

[28] Benestys J, Chen J, Huang Y and Cohen I 2009 Pearson correlation coefficient Noise reduction in speech processing (Springer) pp 1-4

[29] Zhao L, Wei L and Xu C 2016 Physics Letters A 380 654-666

[30] Kenett D Y and Havlin S 2015 Mind 83 Society 14 155-167

[31] Tumminello M, Aste T, Di Matteo T and Mantegna R N 2005 Proceedings of the National Academy of Sciences 102 10421-10426

[32] Sun P G and Yang Y 2013 Physica A: Statistical Mechanics and its Applications 392 1977-1988

[33] China M 20152015 Report on the State of the Environment in China

[34] Fan J, Meng J, Ashkenazy Y, Havlin S and Schellnhuber H J 2017 Proceedings of the National Academy of Sciences 114 7543-7548

[35] Meng J, Fan J, Ashkenazy Y and Havlin S 2017 Chaos: An Interdisciplinary Journal of Nonlinear Science 27035807

[36] Brownlee J 2017 Introduction to time series forecasting with python: how to prepare data and develop models to predict the future (Machine Learning Mastery)

[37] Bloomfield P 2004 Fourier analysis of time series: an introduction (John Wiley \& Sons)

[38] Boers N, Goswami B, Rheinwalt A, Bookhagen B, Hoskins B and Kurths J 2019 Nature 566373

[39] Porter M A, Onnela J P and Mucha P J 2009 Notices of the AMS 56 1082-1097

[40] Fan M, Wong K C, Ryu T, Ravasi T and Gao X 2012 PloS one 7 e39475

[41] Ratti C, Sobolevsky S, Calabrese F, Andris C, Reades J, Martino M, Claxton R and Strogatz S H 2010 PloS one 5 e14248

[42] Tang C, Li X, Cao L and Zhan J 2012 Journal of theoretical biology 306 1-6

[43] Zhang W, Deng W and Li W 2018 Physica A: Statistical Mechanics and its Applications 502 218-227

[44] Yang J, Liu Q, Li X and Cui X 2017 Sustainability 91454

[45] Sun G X, Meharg A A, Li G, Chen Z, Yang L, Chen S C and Zhu Y G 2016 Scientific Reports 6 20953

[46] https://zhuanlan.zhihu.com/p/45131479

[47] Hurst H E 1951 Trans. Amer. Soc. Civil Eng. 116 770-799

[48] Bassingthwaighte J B and Raymond G M 1994 Annals of biomedical engineering 22 432-444

[49] Kirkby M J 1987 Earth Surface Processes and Landforms 12 57-67

[50] Mutua S, Gu C and Yang H 2016 Chaos An Interdisciplinary Journal of Nonlinear Science 26 $441-449$

[51] Stephen M, Gu C and Yang H 2015 PloS one 10 e0143015 